\title{
An insight into the key genes and biological functions associated with insulin resistance in adipose tissue with microarray technology
}

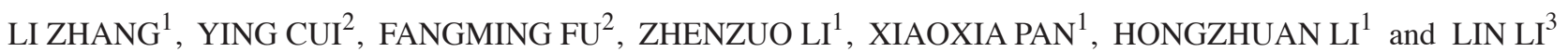 \\ ${ }^{1}$ Department of Endocrinology, The Fourth People's Hospital of Jinan; ${ }^{2}$ Department of Endocrinology, \\ Jinan Central Hospital Affiliated to Shandong University; ${ }^{3}$ Department of Endocrinology, \\ The General Hospital of Jinan Military Command, Jinan, Shandong 250031, P.R. China
}

Received September 9, 2013; Accepted April 8, 2014

DOI: $10.3892 / \mathrm{mmr} .2014 .2909$

\begin{abstract}
In the present study, the key genes and biological functions associated with insulin resistance were investigated by comparing the gene expression profiles of adipose tissue obtained from insulin-sensitive and insulin-resistant patients. The gene expression data set GSE20950 was downloaded from the Gene Expression Omnibus, including 39 adipose tissue samples obtained from insulin-sensitive and insulin-resistant patients undergoing gastric bypass surgery. Adipose samples were divided into two groups (the insulin-sensitive and insulin-resistant groups) and the differentially expressed genes (DEGs) were screened out with packages of $R$. The interactions among DEGs were retrieved with Osprey and functional enrichment analysis was performed with the WebGestalt system. Information regarding the interaction network and enriched biological functions was combined to construct a functional interaction network. Kyoto Encyclopedia of Genes and Genomes (KEGG) pathway analysis was then conducted using the Database for Annotation, Visualization and Integrated Discovery. A total of 170 DEGs were detected in the insulin-sensitive group, 8 downregulated and 162 upregulated. Response to glucose stimulus was the most significantly over-represented functional term. The focal adhesion pathway was identified to be significant in the genes of the functional interaction network. The present study revealed key biological functions and DEGs in adipose tissues associated with insulin resistance, which may facilitate the development of novel therapies for insulin resistance and diabetes.
\end{abstract}

Correspondence to: Dr Lin Li, Department of Endocrinology, The General Hospital of Jinan Military Command, 25 Shifan Road, Tianqiao District, Jinan, Shandong 250031, P.R. China

E-mail: linlidoublel@163.com

Key words: insulin resistance, differentially expressed gene, interaction network, functional enrichment analysis

\section{Introduction}

Diabetes is caused by an absolute or relative deficiency of insulin. It is one of the most prevalent of all chronic diseases, which is characterized by high blood sugar and multiple secondary complications. Obesity is a risk factor for insulin resistance, a precursor of type 2 diabetes, which involves a decreased response to insulin signaling in several peripheral tissues including adipose, liver and muscle $(1,2)$. However, not all obese individuals are insulin-resistant (3).

Adipose tissue secretes numerous hormones and cytokines that function to regulate food intake and nutrient homeostasis, including insulin-like growth factor 2, leptin and resistin (4), and its role in the regulation of metabolism has been increasingly recognized in recent years (5). Previous studies have revealed a number of linkages between obesity and insulin resistance. Obesity-associated chronic inflammation in adipose tissue has a crucial role in the development of obesity-related insulin resistance (6-9). Adipocyte-derived cytokines are important in the pathogenesis of insulin resistance and type 2 diabetes (10). Hirosumi et al (11) indicated that the c-Jun amino-terminal kinase is a crucial mediator of obesity and insulin resistance. However, the molecular mechanisms underlying this effect remain elusive.

In the present study, the gene expression profiles of adipose tissue samples obtained from insulin-sensitive and insulin-resistant patients were compared with the aim of identifying differentially expressed genes (DEGs). Bioinformatic analysis, including interaction network analysis and functional enrichment analysis, were performed to disclose the key biological functions associated with insulin resistance. These findings may advance the understanding of insulin resistance and benefit the development of novel treatment strategies.

\section{Materials and methods}

Microarray data. Microarray data set GSE20950 (12) was downloaded from the Gene Expression Omnibus, including 39 subcutaneous and omental adipose samples, obtained from insulin-sensitive and insulin-resistant obese patients undergoing gastric bypass surgery. The platform was GPL570 
[HG-U133_Plus_2] Affymetrix Human Genome U133 Plus 2.0 Array (Affymetrix, Santa Clara, CA, USA). The probe annotation files were also collected.

Raw data pre-treatment and differential analysis. All of the adipose samples were divided into two groups: The insulin-sensitive group and the insulin-resistant group. Using software $R$, CEL raw expression data were converted into gene expression values, which were then normalized by the median method $(13,14)$. DEGs between the two groups were screened out with the limma package (15) and multiple testing corrections were applied on the P-value with the Benjamin-Hochberg method (16). Only the genes with a false discovery rate $(\mathrm{FDR})<0.05$ and $\log \mathrm{FC}$ (fold change) $\mid>1$ were selected as DEGs.

Interaction network and functional enrichment analysis. The interactions between DEGs were investigated with Osprey (17), which is designed to promote research into protein, protein interactions (PPIs) and protein complexes, and which integrates information from the Biomolecular Interaction Network Database (18) and the General Repository for Interaction Datasets (19), and contains $>50,000$ interactions.

Functional enrichment analysis was applied on the DEGs in the network using WebGestalt $(20,21)$. $\mathrm{P}<0.05$ was set as the threshold.

Functional interaction network and pathway enrichment analysis. Based on the interaction network and functional enrichment analysis result, a functional interaction network was constructed.

Kyoto Encyclopedia of Genes and Genomes (KEGG) (22) pathway analysis was applied on the genes in the functional interaction network using the Database for Annotation, Visualization and Integrated Discovery. $\mathrm{P}<0.05$ was set as the threshold.

\section{Results}

DEGs. The normalized gene expression profiles are revealed in Fig. 1. Differential analysis was performed between the insulin-sensitive and insulin-resistant groups. A total of 170 DEGs were identified in the insulin-sensitive group, of which 8 were downregulated and 162 were upregulated. This result suggested that numerous genes in the adipose tissue obtained from insulin-resistant patients were downregulated and thus contributed to insulin resistance.

Interaction correlation and relevant biological functions. Interactions among DEGs were retrieved using Osprey. In the present study, a total of 33 interactions were obtained (Table I).

Functional enrichment analysis was performed with WebGestalt for all of the gene interactions and eight functional terms were revealed (Table II). Response to glucose stimulus was the most significant functional term and three genes were included; neuronatin (NNAT), transforming growth factor beta receptor II (TGFBR2) and phosphatase and tensin homolog (PTEN).

Functional interaction network and significant pathways. The interaction network was integrated with the functional enrich-
Table I. Interactions between the DEGs.

\begin{tabular}{ll}
\hline Interactor 1 & \multicolumn{1}{c}{ Interactor 2 } \\
\hline TXN & PTEN \\
RAD23B & ZFAND5 \\
NOP16 & GART \\
ITGA2 & FLT4 \\
PIK3CB & FLT4 \\
MMP9 & PIK3CB \\
ETS1 & MMP9 \\
PTEN & PIK3CB \\
ETS1 & MCM8 \\
PTEN & YES1 \\
PTEN & IRS1 \\
IRS1 & LIPE \\
NDUFA7 & ATP5O \\
ZFYVE16 & PIK3CB \\
ITGA2 & COL8A1 \\
AZGP1 & PIP \\
HEYL & MAML1 \\
MMP9 & PTEN \\
NOP16 & POLR3E \\
FZD7 & SFRP1 \\
TGFBR2 & ZFYVE16 \\
ETS1 & PURA \\
TXN & MMP9 \\
MCM8 & PTEN \\
TXN & DLST \\
TXN & ATP5O \\
MCM8 & ANTXR2 \\
AGPAT5 & AGPAT9 \\
IRS1 & PIK3CB \\
DLST & ATP5O \\
NDUFA7 & ENSG00000109390 \\
MCM8 & PURA \\
SMC3 & BCLAF1 \\
& \\
IEN 16
\end{tabular}

DEGs, differentially expressed genes; TGFBR2, transforming growth factor $\beta$ receptor II; PTEN, phosphatase and tensin homolog; PIK3CB, phosphatidylinositol-4, 5-bisphosphate 3-kinase catalytic subunit $\beta$; ITGA2, integrin $\alpha 2$.

ment analysis result to construct the functional interaction network (Fig. 2).

Pathway enrichment analysis revealed that focal adhesion (hsa04510, $\mathrm{P}=0.03695$ ) was over-represented in the genes (Fig. 3). Three DEGs were included in this pathway: Phosphatidylinositol-4, 5-bisphosphate 3-kinase catalytic subunit $\beta$ (PIK3CB), integrin $\alpha 2$ (ITGA2) and PTEN. Focal adhesions are specific types of large macromolecular assemblies through which mechanical force and regulatory signals are transmitted. They serve as the mechanical linkages to the extracellular cell matrix and as a biochemical signaling hub to concentrate and direct numerous signaling proteins at sites of integrin binding and clustering. As a result, focal adhesions 
Table II. Functional terms enriched in DEGs from the interaction network.

\begin{tabular}{|c|c|c|c|}
\hline Term & Count & P-value & Genes \\
\hline GO:0009749 response to glucose stimulus & 3 & 0.000444 & NNAT, TGFBR2, PTEN \\
\hline GO:0006796 phosphate metabolic process & 17 & 0.002208 & $\begin{array}{l}\text { SGK2, PIK3CB, FLT4, NDUFA7, PTPN4, PDK4, } \\
\text { TGFBR2, WNK1, NDUFC1, PTEN, DUSP4, } \\
\text { PDIK1L, DUSP16, BMP2K, ATP5O, YES1, LIPE }\end{array}$ \\
\hline $\begin{array}{l}\text { GO:0048008 platelet-derived growth factor } \\
\text { receptor signaling pathway }\end{array}$ & 3 & 0.008688 & ZFAND5, TIPARP, PTEN \\
\hline GO:0060021 palate development & 3 & 0.023668 & TGFBR2, TIPARP, PYGO2 \\
\hline GO:0046486 glycerolipid metabolic process & 5 & 0.033067 & PIK3CB, AGPAT9, CHPT1, PTEN, LIPE \\
\hline GO:0051272 positive regulation of cell motion & 4 & 0.036903 & ETS1, MMP9, ITGA2, IRS1 \\
\hline GO:0010033 response to organic substance & 11 & 0.042616 & $\begin{array}{l}\text { DUSP4, GATM, NNAT, TGFBR2, ITGA2, } \\
\text { EDEM3, IRS1, PTEN, PGGT1B, TTC3, GART }\end{array}$ \\
\hline $\begin{array}{l}\text { GO:0007167 enzyme linked receptor } \\
\text { protein signaling pathway }\end{array}$ & 7 & 0.042853 & $\begin{array}{l}\text { ZFAND5, FLT4, ZFYVE16, TGFBR2, } \\
\text { TIPARP, IRS1, PTEN }\end{array}$ \\
\hline
\end{tabular}

DEGs, differentially expressed genes. NNAT, neuronatin; TGFBR2, transforming growth factor $\beta$ receptor II; PTEN, phosphatase and tensin homolog; PIK3CB, phosphatidylinositol-4, 5-bisphosphate 3-kinase catalytic subunit $\beta$; ITGA2, integrin $\alpha 2$.

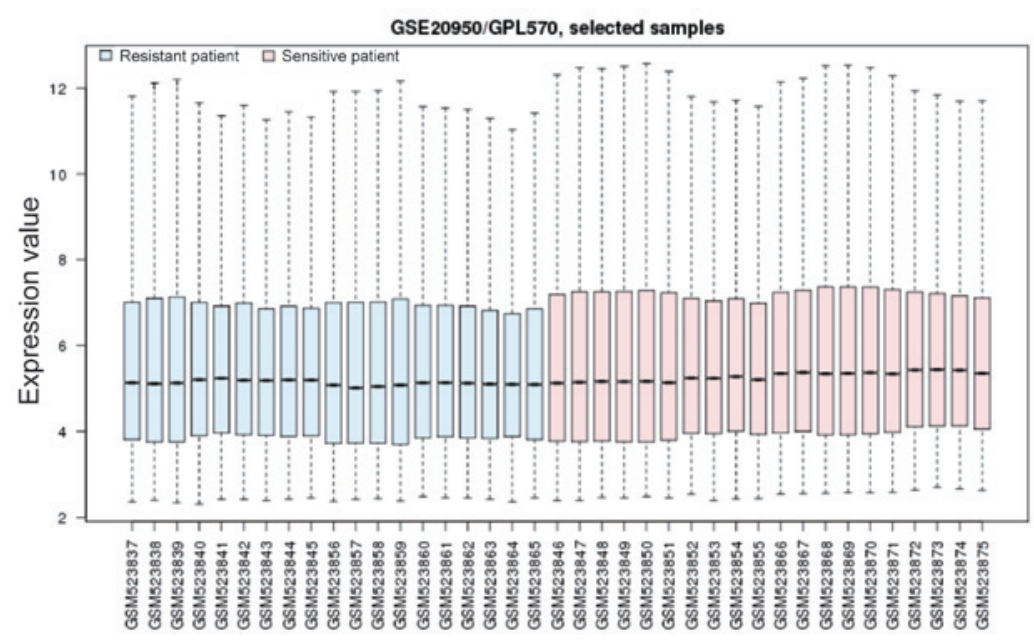

Figure 1. Box plot for normalized gene expression data. Adipose tissue samples from insulin-resistant patients are in blue and those from insulin-sensitive patients are in pink. The medians (black lines) are almost at the same level, indicating a good performance of normalization.

may lead to insulin-resistance and therefore require further investigation.

\section{Discussion}

Adipose tissues have an important role in the development of insulin resistance and thereby contribute to the incidence of type 2 diabetes. However, not all obese individuals exhibit insulin resistance. Therefore, understanding the underlying mechanisms involved is important, as it may benefit the development of novel therapeutic strategies for diabetes treatment. In the present study, the transcriptome of adipose tissues obtained from insulin-sensitive and insulin-resistant patients were compared with the aim of identifying the key DEGs and the associated biological functions. A total of 170 DEGs were obtained, the majority of which were upregulated in the insulin-sensitive group. The interactions among DEGs were retrieved and the functional enrichment analysis on these
DEGs revealed eight significant functional terms, the majority of which were associated with metabolism and signaling pathways. In the KEGG pathway analysis, focal adhesion was identified to be significant.

The results revealed that response to glucose stimulus was the most significant biological function. This was in accordance with the role of adipose tissue in insulin resistance. Three DEGs were included in this term: NNAT, TGFBR2 and PTEN. TGFBR2 has previously been associated with obesity (23). It has been proposed that upregulation of TGFBR2 induced by high extracellular glucose, may contribute to distal tubular hypertrophy in diabetic nephropathy (24). A study by Yang et al (25) demonstrated that TGF- $\beta$ signaling in hepatocytes participates in steatohepatitis through the regulation of cell death and lipid metabolism. PTEN is a key negative regulator of insulin-stimulated glucose uptake in vitro and in vivo (26). Lo et al (27) identified that an increase in PTEN gene expression appears to be associated with the development 


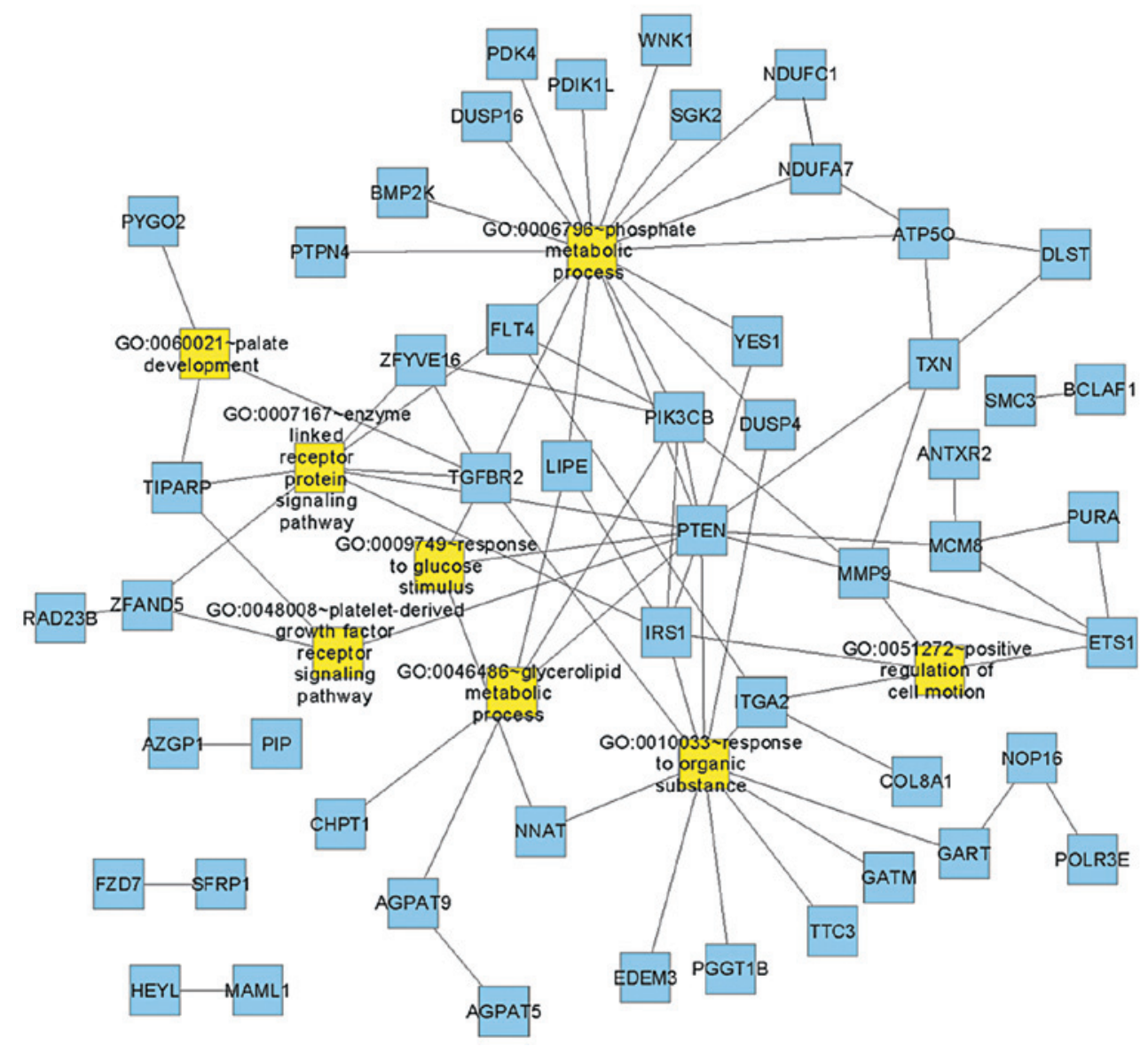

Figure 2. Functional interaction network. Blue rectangles represent differentially expressed genes and the yellow rectangles represent the over-represented functional terms. TGFBR2, transforming growth factor $\beta$ receptor II; PTEN, phosphatase and tensin homolog; PIK3CB, phosphatidylinositol-4, 5-bisphosphate 3-kinase catalytic subunit $\beta$; ITGA2, integrin $\alpha 2$.

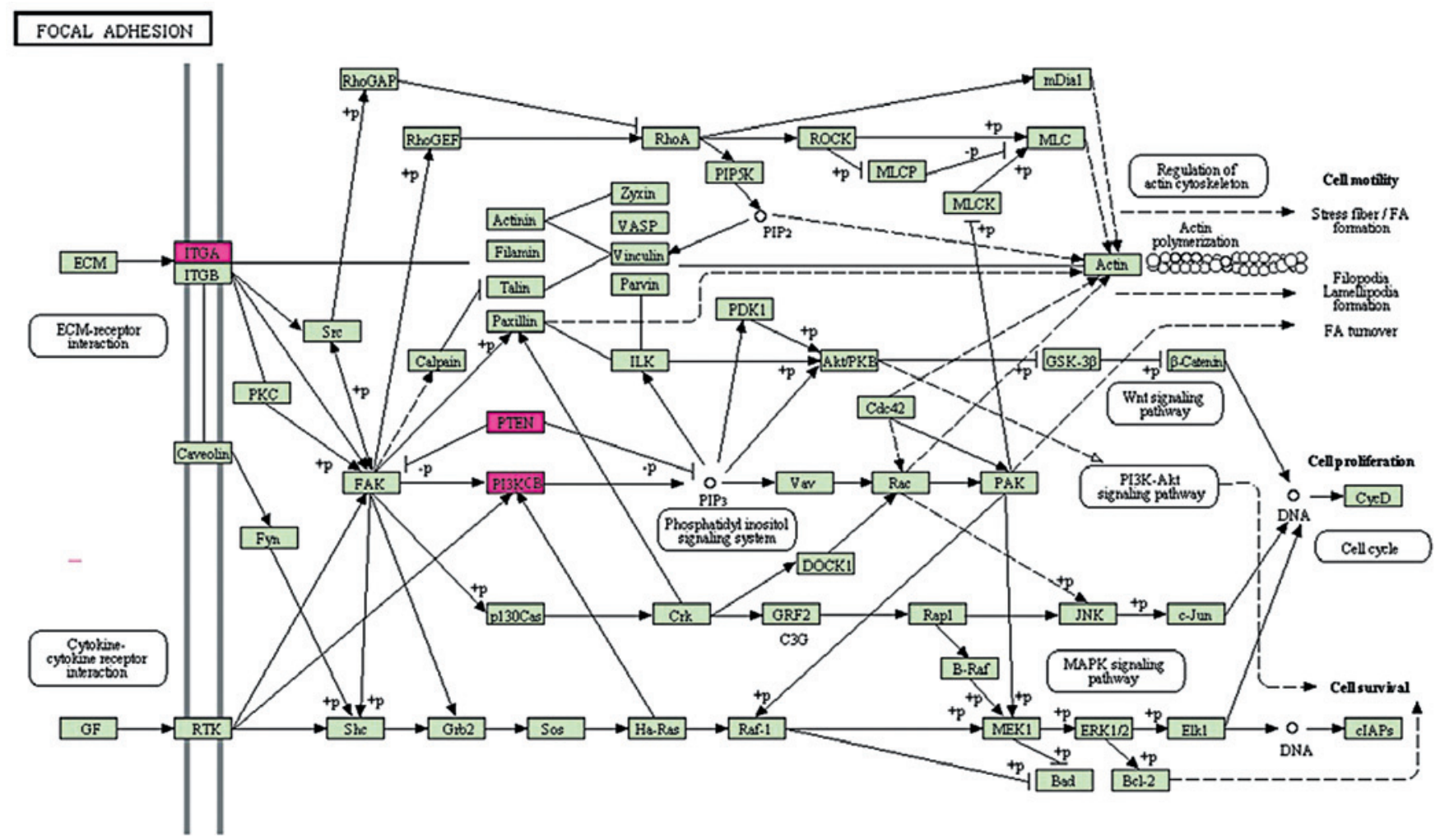

Figure 3. Focal adhesion pathway. Differentially expressed genes are shown in red and the other genes are represented in green. ECM, extracellular matrix; PTEN, phosphatase and tensin homolog; PIK3CB, phosphatidylinositol-4, 5-bisphosphate 3-kinase catalytic subunit $\beta$; ITGA2, integrin $\alpha 2$. 
of insulin resistance. Butler et al (28) found that inhibition of PTEN expression reversed hyperglycemia in diabetic mice. Nakashima et al (29) reported that PTEN negatively regulates insulin signaling in 3T3-L1 adipocytes. Tang et al (30) further indicated that PTEN suppresses insulin signaling through the phosphatidylinositol 3-kinase/Akt pathway in 3T3-L1 adipocytes.

The focal adhesion pathway is closely associated with the insulin signaling pathway. Cell adhesion and focal adhesion kinases regulate insulin receptor substrate-1 expression (31). Baron et al (32) identified that p125Fak focal adhesion kinase is a substrate for the insulin and insulin-like growth factor-I tyrosine kinase receptors. Bisht et al (33) reported that focal adhesion kinase regulates insulin resistance in skeletal muscle. Three DEGs were identified in this pathway, including PIK3CB, ITGA2 and PTEN. PIK3CB is an isoform of the catalytic subunit of phosphoinositide 3-kinase (PI3K), which is important in the signaling pathways involving receptors on the outer membrane of eukaryotic cells. Le Stunff et al (34) identified a variant GATA-binding site in the PIK3CB promoter is a Cis-acting expression quantitative trait locus for this gene and attenuates insulin resistance in obese children, confirming the involvement of this gene in insulin resistance. In another study by Clément et al (35) it was suggested that the cis-regulatory rs361072 promoter variant of PIK3CB is associated with insulin resistance.

In conclusion, the present study described the molecular signatures of adipose tissues obtained from insulin-resistant and insulin-sensitive obese patients. Through comparative analysis, a number of DEGs and relevant biological functions were revealed. These findings provided a theoretical basis and direction for novel strategies for the therapeutic management of diabetes.

\section{References}

1. Kalupahana NS, Moustaid-Moussa N and Claycombe KJ: Immunity as a link between obesity and insulin resistance. Mol Aspects Med 33: 26-34, 2012.

2. Liang CP, Han S, Senokuchi T and Tall AR: The macrophage at the crossroads of insulin resistance and atherosclerosis. Circ Res 100: 1546-1555, 2007.

3. Reaven G: All obese individuals are not created equal: insulin resistance is the major determinant of cardiovascular disease in overweight/obese individuals. Diabetes Vasc Dis Res 2: 105-112, 2005.

4. Fukui $\mathrm{Y}$ and Motojima K: Expression of resistin in the adipose tissue is modulated by various factors including peroxisome proliferator-activated receptor alpha. Diabetes Obes Metab 4: 342-345, 2002

5. Guilherme A, Virbasius JV, Puri V and Czech MP: Adipocyte dysfunctions linking obesity to insulin resistance and type 2 diabetes. Nat Rev Mol Cell Biol 9: 367-377, 2008.

6. Xu H, Barnes GT, Yang Q, et al: Chronic inflammation in fat plays a crucial role in the development of obesity-related insulin resistance. J Clin Invest 112: 1821-1830, 2003.

7. Dandona P, Aljada A and Bandyopadhyay A: Inflammation: the link between insulin resistance, obesity and diabetes. Trends Immunol 25: 4-7, 2004.

8. Shoelson SE, Herrero L and Naaz A: Obesity, inflammation, and insulin resistance. Gastroenterology 132: 2169-2180, 2007.

9. Weisberg SP, Mccann D, Desai M, et al: Obesity is associated with macrophage accumulation in adipose tissue. J Clin Invest 112: 1796-1808, 2003.

10. Greenberg A and Mcdaniel M: Identifying the links between obesity, insulin resistance and beta-cell function: potential role of adipocyte-derived cytokines in the pathogenesis of type 2 diabetes. Eur J Clin Invest 32 (Suppl 3): 24-34, 2002.
11. Hirosumi J, Tuncman G, Chang L, et al: A central role for JNK in obesity and insulin resistance. Nature 420: 333-336, 2002.

12. Hardy OT, Perugini RA, Nicoloro SM, et al: Body mass index-independent inflammation in omental adipose tissue associated with insulin resistance in morbid obesity. Surg Obes Relat Dis 7: 60-67, 2011.

13. Troyanskaya O, Cantor M, Sherlock G, et al: Missing value estimation methods for DNA microarrays. Bioinformatics 17: 520-525, 2001.

14. Fujita A, Sato J, Rodrigues L, Ferreira C and Sogayar M: Evaluating different methods of microarray data normalization. BMC Bioinformatics 7: 469, 2006.

15. Smyth GK: Limma: linear models for microarray data. In Bioinformatics and Computational Biology Solutions using $\mathrm{R}$ and Bioconductor. Gentleman R, Carey V, Dudoit S, Irizarry R and Huber W (eds.). Springer, New York, pp 397-420, 2005.

16. Dudoit S, Shaffer JP and Boldrick JC: Multiple hypothesis testing in microarray experiments. Stat Sci 18: 71-103, 2003.

17. Breitkreutz BJ, Stark C and Tyers M: Osprey: a network visualization system. Genome Biol 4: R22, 2003.

18. Willis RC and Hogue CW: Searching, viewing, and visualizing data in the Biomolecular Interaction Network Database (BIND). Curr Protoc Bioinformatics 8: 8.9, 2006.

19. Breitkreutz B-J, Stark C and Tyers M: The GRID: the general repository for interaction datasets. Genome Biol 4: R23, 2003.

20. Zhang B, Kirov S and Snoddy J: WebGestalt: an integrated system for exploring gene sets in various biological contexts. Nucleic Acids Res 33: W741-W748, 2005.

21. Duncan D, Prodduturi N and Zhang B: WebGestalt2: an updated and expanded version of the Web-based Gene Set Analysis Toolkit. BMC Bioinformatics 11 (Suppl 4): P10, 2010.

22. Kanehisa M, Goto S, Sato Y, et al: KEGG for integration and interpretation of large-scale molecular data sets. Nucleic Acids Res 40: D109-D114, 2012

23. Shoelson SE and Goldfine AB: Getting away from glucose: fanning the flames of obesity-induced inflammation. Nat Med 15: 373-374, 2009.

24. Yang YL, Guh JY, Yang ML, et al: Interaction between high glucose and TGF-beta in cell cycle protein regulations in MDCK cells. J Am Soc Nephrol 9: 182-193, 1998.

25. Yang L, Roh YS, Song J, et al: Transforming growth factor beta signaling in hepatocytes participates in steatohepatitis through regulation of cell death and lipid metabolism. Hepatology 59: 483-495, 2013.

26. Wong J, Kim P, Peacock J, et al: Pten (phosphatase and tensin homologue gene) haploinsufficiency promotes insulin hypersensitivity. Diabetologia 50: 395-403, 2007.

27. Lo YT, Tsao CJ, Liu IM, Liou SS and Cheng JT: Increase of PTEN gene expression in insulin resistance. Horm Metab Res 36: 662-666, 2004 .

28. Butler M, Mckay RA, Popoff IJ, et al: Specific inhibition of PTEN expression reverses hyperglycemia in diabetic mice. Diabetes 51: 1028-1034, 2002.

29. Nakashima N, Sharma PM, Imamura T, Bookstein R and Olefsky JM: The tumor suppressor PTEN negatively regulates insulin signaling in 3T3-L1 adipocytes. J Biol Chem 275: 12889-12895, 2000.

30. Tang X, Powelka AM, Soriano NA, Czech MP and Guilherme A: PTEN, but not SHIP2, suppresses insulin signaling through the phosphatidylinositol 3-kinase/Akt pathway in 3T3-L1 adipocytes. J Biol Chem 280: 22523-22529, 2005.

31. Lebrun P, Baron V, Hauck CR, Schlaepfer DD and Van Obberghen E: Cell adhesion and focal adhesion kinase regulate insulin receptor substrate-1 expression. J Biol Chem 275: 38371-38377, 2000.

32. Baron V, Calléja V, Ferrari P, et al: p125Fak focal adhesion kinase is a substrate for the insulin and insulin-like growth factor-I tyrosine kinase receptors. J Biol Chem 273: 7162-7168, 1998.

33. Bisht B, Goel H and Dey C: Focal adhesion kinase regulates insulin resistance in skeletal muscle. Diabetologia 50: 1058-1069, 2007.

34. Le Stunff C, Dechartres A, Mariot V, et al: Association analysis indicates that a variant GATA-binding site in the PIK3CB promoter is a Cis-acting expression quantitative trait locus for this gene and attenuates insulin resistance in obese children. Diabetes 57: 494-502, 2008.

35. Clément K, Stunff CL, Meirhaeghe A, et al: In obese and non-obese adults, the cis-regulatory rs 361072 promoter variant of PIK3CB is associated with insulin resistance not with type 2 diabetes. Mol Genet Metab 96: 129-132, 2009. 